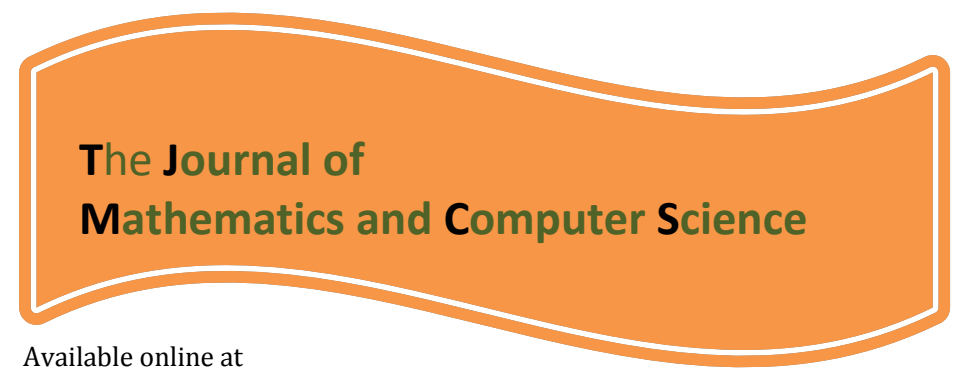

http://www.TJMCS.com

The Journal of Mathematics and Computer Science Vol. 4 No.3 (2012) 473 - 486

\title{
Optimal Control Brain Tumor System with Drog and Its Stability
}

\author{
S.A. Alavi ${ }^{1}$, J. Norabadi ${ }^{2}$, M. Arjmand ${ }^{3}$ \\ 1, 2, 3Dept. of Maths, College ofMathematicsand Computer Science, Hakim Sabzevari University, sabzevar, Iran. \\ 2Javad.nurabadi@gmail.com
}

Received: February 2012, Revised: May 2012

Online Publication: July 2012

\begin{abstract}
It is quite known that there are various methods for treatment of cancer. Although virus therapy has been proved to effective in the improvement of cancer, this method is still at its primary stage. Therefore, treatment methods such as chemotherapy and radiotherapy are still versatile. In these methods, drugs are prescribed. The most important question in the treatment of brain tumors is the rate of drug prescription for the patient so that it can help the patient recover and minimize damages to the healthy cells. A.El-Ghohary demonstrated that a mathematical model of brain tumor system can be seen in an optimal nonlinear control problem. In this paper, attempt is made to transform the nonlinear optimal control problem into an optimal control problem in the measure theory and to approximate a new problem with a linear programming problem and subsequently, to specify the drug dose for the patients with cancer. In addition, we deal with the examination of stability of system balance points. Using drug dose control stabilizes the unstable balance points of the tumor system. In the end, a comparison is made between the results obtained from the above mentioned method and the approximate solution proposed by Al-Gohary.
\end{abstract}

Keywords: Nonlinear optimal control, Measure theory, Tumor, linear programming.

\section{Introduction}

Cancer means growth, multiplication and sometimes abnormal proliferation of cells in the human body which is the second major cause of death around the world and an estimated number of $8,500,000$ people die every year. Surgical operation, chemotherapy, hormone therapy and radiotherapy are considered the effective ways of cancer treatment. Any of the specific methods mentioned above are used based on the kind, stage and location of cancer. Human body is formed by millions of cells which, altogether, build tissues such as muscles, bones and skin. In response to the stimuli, most natural human cells penetrate from the 
inside and outside of the body and grow and duplicate and finally die. If this process happens in balance and under its proper condition, body remains healthy and keeps on performing in a natural order. But problems occur when some genes of a natural cell suffer mutation and the cell transforms into a cancer cell. In cancer, the cells lose their ability to divide and grow normally and this issue results in the capture, destruction and decay of the healthy tissues. These cancer cells are accumulated and then destroy the healthy cells of the tissues and create a mass called tumor.

If the tumor is restricted by a limited layer and doesn't permeate the other tissues and organs the tumor is called "benign" (non-cancerous) and if the tumor is extended or potentially spreads and encompasses other tissues and organs is called "malignant" or cancerous.

Different mathematical models have been presented for tumor, the model proposed in this paper have been proposed in 2006 by Rassel and Dinjelli[3], then it has been revised and modified by Al-Gohary [1].it will be demonstrated that a mathematical model for the treatment of cancer through drug prescription is an optimal control problem with nonlinear systems which using measure theory it has been transformed into a linear optimization problem and solved.

\section{Tumor model}

This section concerns with the construction of the mathematical model of tumor system with drug. Also the linear stability analysis of this model is introduced. The tumor system with drug will be constructed based on the following processes. The growth of immune cells can be stimulated by the presence of tumor cells, and these immune cells destroy the tumor cells through a kinetic process. It should be noted that the presence of a detectable tumor does not necessarily imply that the tumor has completely escaped from active immunosurveillance. It is completely possible that although a tumor is immunogenetic, the immune response is not sufficient on its own to entirely combat the rapid growth of the tumor population and the final development into a tumor. Actually, there is even some speculation that all tumors are immunogenetic[7]. The normal cells and tumor cells compete for available resources, while immune cells and tumors cells compete in a prey-predator behavior. Finally, the optimal drug therapies are calculated from the conditions that minimize both the final tumor population cells, the average tumor over time, while keeping the normal cells above a requested level. For more details about the kinetics processes of this model can be found in Refs. [8-10]. we start with the building of the tumor with drug mathematical model.

Assume that $I(t)$ denote the number of immune cells at time t, while $T(t)$ is the number of tumor cells at same time $t$ and $H(t)$ is the number of the host cells or normal cells at the same time $t$.

We consider that the resource of the immune cells is to be outside the system so it is reasonable to assume that theimmune cells have a constant influx rate $s$. In the absence of any tumor, the cells will die off at a per capita rate $d 1$,resulting in long-term population size of $s / d 1$ cells. Therefore, immune cell proliferation will suffer from immunity uponimmune crowding. The immune response stimulates by positive nonlinear growth in the presence of tumor cells That is

$$
\frac{r_{3} I(t) T(t)}{\alpha+T(t)}
$$


Where $r_{3}$ and a are positive real constants. Moreover, the reaction of immune cells and tumor cells represents in either death of tumor cells or the inactivation of the immune cells which can be represented by the following two competition terms:

$$
\frac{d I}{\mathrm{dt}}=-\mathrm{c}_{1} \mathrm{~T}(\mathrm{t}) \mathrm{I}(\mathrm{t}), \quad \frac{d T}{d t}=-c_{2} T(t) I(t)
$$

where $c_{1}$ and $c_{2}$ are also positive real constants.

Now, the tumor cells as well as hosting cells can be modelled by a logistic growth law with parameters $r_{1}$ and $b_{1}$, representing the per capita growth rate and reciprocal carrying capacity of the tumor cells, $r_{2}$ and $b_{2}$ represent the same rates for the host cells. In addition, there are two terms which represent the competition between tumor and host cells. Based on the above description of the model, all the terms together give the following system of nonlinear ordinary differential equations:

$$
\begin{aligned}
& \frac{\mathrm{dT}}{d t}=\mathrm{T}(\mathrm{t})\left[\mathrm{r}_{1}\left(1-\mathrm{b}_{1} \mathrm{~T}(\mathrm{t})\right)-\mathrm{c}_{2} \mathrm{I}(\mathrm{t})-\mathrm{c}_{3} \mathrm{H}(\mathrm{t})\right] \\
& \frac{d H}{d t}=H(t)\left[r_{2}\left(1-b_{2} H(t)\right)-c_{4} T(t)\right] \\
& \frac{d I}{d t}=s+I(t)\left[\frac{r_{3} T(t)}{\alpha+T(t)}-c_{1} T(t)-d_{1}\right.
\end{aligned}
$$

This model describes the tumor model without drug interaction [11]. Next, we will extend the mathematical model (3) to describe the dynamical behavior of the tumor system with drug interaction.Now we add the effect of the drug on the tumor system. We denote he amount of drug at the tumor site at time $t$ by $D(t)$. Also we assume that the drug kills all types of cells, but that the kill rate differs for every type of cell, with theresponse curve in all the cases given by an exponential

$$
\varphi(D)=\left(1-\mathrm{e}^{-\mathrm{kD}}\right)
$$

where $\varphi(D)$ is the fractional cell kill for a given amount of $\operatorname{drug} D$, at the tumor site and $k$ is positive real constant.

We denote the response system coefficients by $a_{1} ; a_{2}$ and $a_{3}$. Now let us add these terms to the system (3) as well as anequation for $D(t)$, the amount of drug at the tumor site. This can be determined by the dose given $v(t)$, and a per capitadecay rate of the drug once it is injected. This decay rate incorporates all the pathways of elimination of the drug.Therefore, tumor system with drug interaction can describe the following system of nonlinear differential equations

$$
\begin{aligned}
& \dot{T}(\mathrm{t})=\mathrm{T}(\mathrm{t})\left[\mathrm{r}_{1}\left(1-\mathrm{b}_{1} \mathrm{~T}(\mathrm{t})\right)-\mathrm{c}_{2} \mathrm{I}(\mathrm{t})-\mathrm{c}_{3} \mathrm{H}(\mathrm{t})-\alpha_{1}\left(1-e^{-D(t)}\right)\right] \\
& \dot{H}(t)=H(t)\left[r_{2}\left(1-b_{2} H(t)\right)-c_{4} T(t)-\alpha_{2}\left(1-e^{-D(t)}\right)\right](5) \\
& \dot{I}(t)=s+I(t)\left[\frac{r_{3} T(t)}{\alpha+T(t)}-c_{1} T(t)-d_{1}-\alpha_{3}\left(1-e^{-D(t)}\right)\right] \\
& \dot{\mathrm{D}}(\mathrm{t})=v(\mathrm{t})-\mathrm{d}_{2} \mathrm{D}(\mathrm{t})
\end{aligned}
$$

Where $d_{2}$ is per capita death rate of the drug amount. Next we look at the linear stability analysis of both tumor and tumor with drug systems. Also, we study the optimal control of the 
tumor system with drug using the dose $v(t)$ as control variable and tumor, host, immune cells and drug as state variables.

\section{Reduction of the system parameters}

In this subsection, we will reduce the system parameters by defining new variables and new system parameters. Now, the following new variables are used to reduce the number of the system parameters from 16 to 12 only:

$x_{1}=b_{1} T(t), \quad x_{2}=b_{2} H(t), \quad x_{3}=\frac{d_{2}}{s} I(t), \quad v(t)=d_{2} u(t), \quad x_{4}=D(t), \quad \tau=d_{2} t$

where the new system parameters are related to old system parameters by the following relations:

$\dot{x_{1}}=x_{1}\left[k_{1}\left(1-x_{1}\right)-n_{2} x_{3}-n_{3} x_{2}-m_{1}\left(1-e^{-x_{4}}\right)\right]$

$\dot{x_{2}}=x_{2}\left[k_{2}\left(1-x_{2}\right)-n_{4} x_{1}-m_{2}\left(1-e^{-x_{4}}\right)\right]$

$\dot{x_{3}}=1+x_{3}\left[\frac{k_{3} x_{1}}{v_{1}+x_{1}}-n_{1} x_{1}-v_{2}-m_{3}\left(1-e^{-x_{4}}\right)\right]$

$\dot{x_{4}}=u-x_{4}$.

where the new system parameters are related to old system parameters by the following relations:

$\left\{\begin{array}{ccc}k_{i}=\frac{r_{i}}{d_{2}}, & m_{i}=\frac{\alpha_{i}}{d_{2}}, \quad(i=1,2,3) \quad n_{1}=\frac{c_{1}}{d_{2} b_{1}} & v_{2}=\frac{d_{1}}{d_{2}} \\ n_{2}=\frac{c_{2} s}{d_{2}^{2}}, & n_{3}=\frac{c_{3}}{b_{2} d_{2}}, \quad n_{4}=\frac{c_{4}}{b_{1} d_{2}}, & v_{1}=\alpha b_{1}\end{array}\right.$

The system (7) is more simple than (5) for the mathematical study since the number of system parameters has been reduced from 16 to 12 only.

\section{Stability of the equilibrium states}

The present section is devoted to study the linear stability of the tumor system whit drog.

(1) The first equilibrium state is given by $E_{1}=\left(0,0, \frac{1}{v_{2}}, 0\right)$. The eigenvalues of the Jacobian matrix about this position are

$$
\begin{gathered}
\gamma_{1}=k_{1}-\frac{n_{2}}{v_{2}} \\
\gamma_{2}=k_{2} \\
\gamma_{3}=-v_{2} \\
\gamma_{4}=-1
\end{gathered}
$$

It is to verify whether two of these eigenvalues are positive if $k_{1}>\frac{n_{2}}{v_{2}}$ so this equilibriumpoint is absolutely unstable. 
(2)The second equilibrium state is given by $E_{2}=\left(0,1, \frac{1}{v_{2}}, 0\right)$. The eigenvalues of the Jacobian about this position are

$$
\begin{gathered}
\gamma_{1}=k_{1}-\frac{n_{2}}{v_{2}} \\
\gamma_{2}=-k_{2} \\
\gamma_{3}=-v_{2} \\
\gamma_{4}=-1
\end{gathered}
$$

It is easy to verify that the system is absolutely unstable if $k_{1}>\frac{n_{2}}{v_{2}}$.

(3) The third equilibrium state is given by $E_{3}=\left(a, 1, \frac{k_{1}(1-a)}{n_{2}}, 0\right)$, where the values of a are the real roots of the cubic equation:

$$
\begin{gathered}
n_{1} n_{2}^{2} x_{1}^{3}+\left(v_{2}+2 n_{1}+n_{1} v_{1}-k_{3}\right) n_{2} k_{1} x_{1}^{2}+\left(n_{1} k_{1}-n_{2}-k_{1} k_{3}+v_{1} v_{2} k_{1}+n_{1} v_{1} k_{1}+v_{2} k_{1}\right) k_{1} x_{1} \\
-k_{1}^{2}\left(1+v_{1}\right)=0
\end{gathered}
$$

which has exact three roots giving three equilibrium states; among them at least one real root yields at a real equilibrium state and the eigenvalues of the Jacobian about this state are

$$
\begin{gathered}
\gamma_{1}=k_{2}-n_{4} a \\
\gamma_{2}=\frac{n_{2}(\delta-1)-k_{1}^{2} a(1-a)}{2 k_{1}(1-a)} \\
\gamma_{3}=\frac{n_{2}(-\delta-1)-k_{1}^{2} a(1-a)}{2 k_{1}(1-a)} \\
\gamma_{4}=-1
\end{gathered}
$$

Where

$\delta=\left\{\left(\mathrm{n}_{2}^{2}+\mathrm{k}_{1}^{4} \mathrm{a}^{2}(1-\mathrm{a})^{2}+2 \mathrm{k}_{1}^{2} \mathrm{n}_{2} \mathrm{a}(1-\mathrm{a})+4 k_{1}^{3} a(1-a)^{3}\left[n_{1}\left(v_{1}+a\right)^{2}-k_{3} v_{1}\right]\right) / n_{2}^{2}\left(v_{1}+a\right)\right\}^{\frac{1}{2}}$

Since, the first eigenvalue of the Jacobian can be positive or negative depending upon the value of the parameters $\mathrm{k}_{2}, n_{4}$, so this equilibrium state is absolutely unstable if $\mathrm{k}_{2}>\mathrm{n}_{4}$ aand further the stability analysis of this equilibrium state depends on the two other eigenvalues.

(4) The fourth equilibrium state of the tumor system with drug is given by $E_{4}=\left(0,0, \frac{1}{v_{2}+m_{3}\left(1-e^{-d}\right)}, d\right)$ where $x_{4}=d=u \neq 0$ which is absolutely unstable if

$$
k_{1}+k_{2}>\left(m_{1}+m_{2}\right)\left(1-e^{-d}\right)-n_{2}\left[v_{2}+m_{3}\left(1-e^{-d}\right)\right]^{-1}
$$

(5)The fifth equilibrium state of the tumor system with drug is given by $E_{5}=\left(0, \frac{k_{2}-m_{2}\left(1-e^{-d}\right)}{k_{2}}, \frac{1}{v_{2}+m_{3}\left(1-e^{-d}\right)}, d\right)$ where $x_{4}=d=u \neq 0$, which is absolutely unstable if

$$
k_{1}+k_{2}>\left(m_{1}+m_{2}\right)\left(1-e^{-d}\right)-n_{2}\left[v_{2}+m_{3}\left(1-e^{-d}\right)\right]^{-1}+n_{3} m_{2}\left(1-e^{-d}\right) / k_{2}
$$

\section{Optimal drug for the tumor}


In this section, we will study the problem of optimal control of the tumor system with drug. We consider the effect of chemotherapy to our tumor system. For the purpose of optimal control, we will use Pontryagin minimum principle and measure theory. Since the state of the tumor system with drug is given by $x_{i}$ with equilibrium states $x_{i}=\bar{x}_{i} ; \quad(\mathrm{i}=1,2,3,4)$. If $u=u(t)$ is

the control variable, state equations that typifies potentially unstable behavior are

$$
\begin{gathered}
\dot{x_{1}}=x_{1}\left[k_{1}\left(1-x_{1}\right)-n_{2} x_{3}-n_{3} x_{2}-m_{1}\left(1-e^{-x_{4}}\right)\right] \\
\dot{x_{2}}=x_{2}\left[k_{2}\left(1-x_{2}\right)-n_{4} x_{1}-m_{2}\left(1-e^{-x_{4}}\right)\right] \\
\dot{x_{3}}=1+x_{3}\left[\frac{k_{3} x_{1}}{v_{1}+x_{1}}-n_{1} x_{1}-v_{2}-m_{3}\left(1-e^{-x_{4}}\right)\right] \\
\dot{x_{4}}=u-x_{4}
\end{gathered}
$$

without any amount of drug, the tumor system with drug is absolutely unstable. The target of this section to be reached in a given time $T$ is the equilibrium state with an optimal drug. Hence, the initial and final conditions are

$x_{i}(0)=x_{i 0}, x_{i}(T)=\bar{x}_{i} i=1,2,3,4$

Next, we select the cost function as the one that penalizes the usage with large magnitude and define the cost by

$$
J=\frac{1}{2} \int_{0}^{T}\left[\beta(u-\bar{u})^{2}+\sum_{i=1}^{4} \beta_{i}\left(x_{i}-\bar{x}_{i}\right)^{2}\right] d t,
$$

Where $\beta, \beta_{i},(i=1,2,3,4)$,are positive constants.

In thenext sectiontheanalytical solution, using the Pontryagin minimum principle andinthe end, weusemeasure theoryto solve it.

\subsection{Solution of the problem using Pontryagin minimum principle}

We now go through the procedure of applying Pontryagin minimum principle. First, we replace the cost integral (10) by an additional state variable $x(t)$, which satisfies the state equation

$\dot{x}=\frac{1}{2}\left[\beta(u-\bar{u})^{2}+\sum_{i=1}^{4} \beta_{i}\left(x_{i}-\bar{x}_{i}\right)^{2}\right]$

Next, we introduce the five co-state variable $\rho_{i},(i=0,1,2,3,4)$ and so the Hamiltonian function is given by

$H=\rho_{0} \dot{x}+\sum_{i=1}^{4} \rho_{i} \dot{x}_{i}$

Substituting (9) and (11) in (12) we get

$H=\rho_{0}\left[\beta(u-\bar{u})^{2}+\sum_{i=1}^{4} \beta_{i}\left(x_{i}-\bar{x}_{i}\right)^{2}\right] / 2+\rho_{1} x_{1}\left[k_{1}\left(1-x_{1}\right)-n_{2} x_{3}-n_{3} x_{2}\right]+\rho_{2} x_{2}\left[k_{2}(1-\right.$

$\left.\left.x_{2}\right)-n_{4} x_{1}\right]+\rho_{3} x_{3}\left[\frac{k_{3} x_{1}}{v_{1}+x_{1}}-n_{1} x_{1}-v_{2}\right]+\rho_{4}\left(u-x_{4}\right)-\left(1-e^{-x_{4}}\right) \sum_{i=1}^{3} \rho_{i} m_{i} x_{i}+\rho_{3}$,

The Hamilton equations are 
$\dot{\rho}_{0}=-\frac{\partial H}{\partial x}=0, \quad \dot{\rho}_{i}=\frac{\partial H}{\partial x_{i}}, \quad \frac{\partial H}{\partial u}=0 \quad i=1,2,3,4$

The other pair of Hamilton equations are just the state Esq. (9) and (11). Without the loss of generality, we can choose $\rho_{0}=-1$. Substituting (13) in (14), the co-state equations can be derived in the following form:

$$
\begin{aligned}
& +m_{1} \rho_{1}\left(1-e^{-x_{4}}\right), \\
& \dot{\rho}_{2}=\gamma_{2}\left(x_{2}-\bar{x}_{2}\right)+n_{3} x_{1} \rho_{1}-\rho_{2}\left[k_{2}\left(1-2 x_{2}\right)-n_{4} x_{1}\right]+m_{2} \rho_{2}\left(1-e^{-x_{4}}\right),(15) \\
& \dot{\rho}_{3}=\gamma_{3}\left(x_{3}-\bar{x}_{3}\right)+n_{2} x_{1} \rho_{1}-\rho_{3}\left[k_{3} x_{1} /\left(v_{1}+x_{1}\right)-n_{1} x_{1}-v_{2}\right]+m_{3} \rho_{3}\left(1-e^{-x_{4}}\right), \\
& \dot{\rho}_{4}=\gamma_{4}\left(x_{4}-\bar{x}_{4}\right)+\rho_{4}+\left(m_{2} x_{2} \rho_{2}+m_{1} x_{1} \rho_{1}+m_{3} x_{3} \rho_{3}\right) e^{-x_{4},},
\end{aligned}
$$

The control function that has to be used is determined from the condition $\frac{\partial H}{\partial u}=0$. Hence, we get

$$
u=\bar{u}+\frac{\rho_{4}}{\gamma}
$$

Substituting (16) in (9), we get the nonlinear controlled state system

$$
\begin{gathered}
\dot{x_{1}}=x_{1}\left[k_{1}\left(1-x_{1}\right)-n_{2} x_{3}-n_{3} x_{2}-m_{1}\left(1-e^{-x_{4}}\right)\right] \\
\dot{x_{2}}=x_{2}\left[k_{2}\left(1-x_{2}\right)-n_{4} x_{1}-m_{2}\left(1-e^{-x_{4}}\right)\right] \\
\dot{x_{3}}=1+x_{3}\left[\frac{k_{3} x_{1}}{v_{1}+x_{1}}-n_{1} x_{1}-v_{2}-m_{3}\left(1-e^{-x_{4}}\right)\right] \\
\dot{x_{4}}=-x_{4}+\bar{u}+\rho_{4} / \gamma,
\end{gathered}
$$

This system of nonlinear differential equations form a complete system to solve the optimal control of the tumor system with drug. This system has the following boundary conditions:

$$
x_{i}(0)=x_{i 0}, x_{i}(T)=\bar{x}_{i}, \rho_{i}(T)=0 \quad i=1,2,3,4 .
$$

Next, we look at the numerical integration of the nonlinear systems (17) with the boundary conditions (18) using Runge-Kutta numerical method with step size 0.01.

\section{2. approximate solution of the problem using Measure Theory}

Assume:

$$
\begin{gathered}
x(t)=\left(x_{1}(t), x_{2}(t), x_{3}(t), x_{4}(t)\right), \\
f(t, x, u)=\left[\beta(u-\bar{u})^{2}+\sum_{i=1}^{4} \beta_{i}\left(x_{i}-\bar{x}_{i}\right)^{2}\right] / 2 \\
g_{1}(t, x, u)=x_{1}\left[k_{1}\left(1-x_{1}\right)-n_{2} x_{3}-n_{3} x_{2}-m_{1}\left(1-e^{-x_{4}}\right)\right] \\
g_{2}(t, x, u)=x_{2}\left[k_{2}\left(1-x_{2}\right)-n_{4} x_{1}-m_{2}\left(1-e^{-x_{4}}\right)\right]
\end{gathered}
$$




$$
\begin{gathered}
g_{3}(t, x, u)=1+x_{3}\left[\frac{k_{3} x_{1}}{v_{1}+x_{1}}-n_{1} x_{1}-v_{2}-m_{3}\left(1-e^{-x_{4}}\right)\right] \\
g_{4}(t, x, u)=u-x_{4} \\
g(t, x, u)=\left(g_{1}(t, x, u), g_{2}(t, x, u), g_{3}(t, x, u), g_{4}(t, x, u)\right), \\
x(0)=x_{0}=\left(x_{10}, x_{20}, x_{30}, x_{40}\right), \quad \mathrm{U}=[a, \mathrm{~b}], \quad x(T)=\bar{x}=\left(\bar{x}_{1}, \bar{x}_{2}, \bar{x}_{3}, \bar{x}_{4}\right), \quad J=[0, T],
\end{gathered}
$$

Also $\mathrm{A}$ as a cell in $R^{4}$, take:

$\forall t \in J \quad x(t) \in A$.

In this optimal control problem (19), it can totally be written as:

$\operatorname{Min} \int_{0}^{T} f(t, x, u) d t$

$$
\text { s.t: } x^{\prime}=g(t, x, u) \quad t \in J=[0, T](20)
$$

$x(0)=x_{0}, \quad x(T)=\bar{x}_{i} \quad u \in U$,

Where $f$ and $g$ are continuous functions over $Q=J \times A \times U$.

Assume that the control function $u(t): J \rightarrow U$ is measureable, $x($.$) is called a u($.$) circuit$ Whereas it is absolutely continuous and is applicable to the control system (20). Hence, control-circuit couples $S()=.(x(),. u()$.$) is called an admissible couple. If the u$ is the control function and $x$ is a circuit for $u$ that $x(0)=x_{0}$ and $x(T)=\bar{x}$, the total admissible couples are demonstrated by $W$. function $I: W \rightarrow R$ is defined as follows:

$I(s)=\int_{J} f(t, x, u) d t$

Therefore, classic optimal control problem (20) is transformed into optimal control problem:

Min $I(s)$

$$
\text { s.t: } s \in W
$$

Now the optimal control problem above is transformed into an optimal control problem in the measure theory. Assume $B$ is an open set in $R^{5}$ including $A \times J$ and $C^{1}(B)$ are the collection of all the continuous function derivatives over $B$ which in itself and their partial derivatives of the first rank on $B$ are bounded, for each $\varphi \in C^{1}(B)$ the function of $\varphi^{g}$ over the $Q$ is defined as follows:

$$
\varphi^{g}(t, x, u)=\varphi_{x}(t, x) g(t, x, u)+\varphi_{t}(t, x)(22)
$$

Therefore, we have:

$$
\int_{J} \varphi^{\mathrm{g}}(\mathrm{t}, \mathrm{x}, \mathrm{u}) \mathrm{dt}=\varphi\left(\mathrm{t}_{\mathrm{b}}, \mathrm{x}_{\mathrm{b}}\right)-\varphi\left(\mathrm{t}_{\mathrm{a}}, \mathrm{x}_{\mathrm{a}}\right)=\Delta \varphi,
$$

In this particular state if $h(t, x, u)$ is a real continuous function over the $Q$ so that it is just time-dependent: 


$$
\int_{J} h(t, x, u) d t=a_{h}
$$

The constant number $a_{h}$ is theLebesgue integral value of the function hover $J$.

Now, we assume that $J^{0}$ is the internal points of $J$ and $D\left(J^{0}\right)$ is the space of all the real functions of infinite derivative compact base in $J$ the functions $\psi_{j} j=1,2,3$, and $4, \psi_{j}$ over $Q$ is defined as follows:

$\psi_{j}(t, x, u)=x_{j} \Phi^{\prime}(t)+g_{j}(t, x, u) \Phi(t) \quad \forall \Phi \in D\left(J^{0}\right)$

Where $g_{j}$ and $x_{j}$ for $j=1,2,3,4$ are the components of the function $g$ and $x$ in the equation (19). In this case using integration by parts we have:

$\int_{\mathrm{J}} \psi_{j}(t, x, u) d t=0, \quad j=1,2,3,4(25)$

Equations 23-25 show the properties of the admissible couples. Such as the admissible couple $s$, we have:

$$
\Lambda_{s}: F \rightarrow \int_{J} F(t, x, u) d t
$$

A linear positive functional is over space $C(Q)$, the $C(Q)$ is the space of continuous real functions on $Q$. hence, for each acceptable couple $s$, there is a positive linear functional $\Lambda_{s}$ which is applicable to the following properties:

$$
\begin{gathered}
\Lambda_{s}\left(\varphi^{g}\right)=\Delta \varphi \quad \varphi \in C^{1}(B) \\
\Lambda_{s}(h)=a_{h} \quad h \in C_{1}(Q) \\
\Lambda_{s}\left(\psi_{j}\right)=0 \quad \psi_{j} \in D\left(J^{0}\right), \quad j=1,2,3,4
\end{gathered}
$$

Where $C_{1}(Q)$ is a sub space of $C(Q)$ which these sets of functions are time dependent.

Assume that $W_{1}$ is the set of all the linear positive functions over $C(Q)$ which is applicable to the properties mentioned above. According to the Reiss theorem, there is corresponding positive measure for each function over $Q$ which is applicable to the following conditions:

$$
\begin{aligned}
& \mu\left(\varphi^{g}\right)=\Delta \varphi \varphi \in C^{1}(B) \\
& \mu(h)=a_{h} \quad h \in C_{1}(Q) \\
& \mu\left(\psi_{j}\right)=0 \quad \psi_{j} \in D\left(J^{0}\right), \quad j=1,2,3,4
\end{aligned}
$$

The total measures which are applicable to the conditions above are shown by $\mathrm{W}_{1}$. Thus, choosing the linear function $\varphi: W_{1} \rightarrow R$ and inserting in the following relation:

$$
\varphi(\mu)=\int_{Q} f d \mu=\mu(f)
$$


Classic optimal control problem is transformed into a new optimization problem in the measure theory, i.e. minimum of function $\varphi$ over $\mathrm{W}_{1}$, so we have:

\section{Theorem:}

optimal problem in the measure theory, i.e.:

$$
\begin{aligned}
& \operatorname{Min} \quad \varphi(\mu) \\
& \text { s.t: } \mu \in W_{1}
\end{aligned}
$$

has an answer such as $\mu^{0} \in W_{1}$ (proof in [6]).

Obviously, the solution of the classic optimal problem, i.e. $\mu^{0}(f)$, is the same solution as the main optimal problem. For the calculation of its approximate value, we do as follows:

Primarily, we divide the length of each interval related with components $t, x_{1}, x_{2}, x_{3}, x_{4}, u$ to a number of equal sub intervals, in this case $Q$ is partitioned to sub cell $Q_{j},(j=1,2, \ldots, n)$. We choose point $Z_{j}=\left(t_{j}, x_{1 j}, x_{2 j}, x_{3 j}, x_{4 j}, u_{j}\right)$ in the region $Q_{j}$ and assume $\sigma=\left\{Z_{j} ; j=\right.$ $1,2, \ldots, n\}$, in this case $\sigma$ is a density subset in $Q$. it can be proved that through choosing constant natural numbers $N, M_{1}, M_{2}$, and $M_{3}$ the approximate value $\mu^{0}(f)$ is the solution to the linear planning problem with finite dimension [6]:

$\operatorname{Min} \quad \sum_{j=1}^{N} \alpha_{j} f\left(z_{j}\right)$

$$
\begin{array}{cc}
\text { s.t: } \sum_{j=1}^{N} \alpha_{j} \varphi_{i}^{g}\left(z_{j}\right)=\Delta \varphi_{i} & i=1,2, \ldots, M_{1}, \\
\sum_{j=1}^{N} \alpha_{j} h_{i}\left(t_{j}\right)=a_{i} & i=1,2, \ldots, M_{2}, \\
\sum_{j=1}^{N} \alpha_{j} \psi_{k i}\left(z_{j}\right)=0 & i=1,2, \ldots, M_{3}, \quad k=1,2,3,4 \\
\alpha_{j} \geq 0, & j=1,2, \ldots, N
\end{array}
$$

In which $a_{i}=\int_{J} h_{i}(t) d t, i=1,2, \ldots, M 2$.

For more simplification in the calculations functions $\varphi_{i}, \mathrm{~h}_{\mathrm{i}}, \psi_{k i}$ can be chosen as follows:

$$
\Phi(i)=\left\{\begin{array}{rl}
x_{1}^{k} & i=4 k-3 \\
x_{2}^{k} & i=4 k-2 \\
x_{3}^{k} & i=4 k-1 \\
x_{4}^{k} & i=4 k
\end{array} \quad i=1,2, \ldots, M_{1},\right.
$$




$$
h_{i}(t)=\left\{\begin{array}{cc}
1 & t \in\left(\frac{i-1}{M_{2}}, \frac{i}{M_{2}}\right) \\
0 & \text { o. w }
\end{array} \quad i=1,2, \ldots, M_{2},\right.
$$

Also, functions $\Phi_{i}$ which is used for definition of functions $\psi_{k i}$ can be chosen as follows:

$$
\Phi_{i}= \begin{cases}\sin (2 k \pi t) & i=4 k-3,4 k-2 \\ 1-\cos (2 k \pi t) & i=4 k-1,4 k\end{cases}
$$

Assuming $M=M_{1}+M_{2}+2 M_{3}$, linear planning problem containing $N$ the variable $M$ is unknown and solving it and calculating the coefficients $\alpha_{j}$ the approximate control function can be proves using the method proposed by Robio [6].

\section{Numerical solution}

In this part nonlinear optimal control problem can be solved using the methods already mentioned and the diagrams of optimal values of tumor cells, healthy cells, immune cells, optimal dosage of drug in the tumor system with drug, in terms of primary variables and the proposed parameters are obtained:

$$
\begin{gathered}
\mathrm{k}_{1}=30, \mathrm{k}_{2}=48, \quad \mathrm{k}_{3}=29, \quad \mathrm{n}_{1}=2, \mathrm{n}_{2}=1.3, \mathrm{n}_{3}=0.47, \mathrm{n}_{4}=8, \mathrm{~m}_{1}=9, \quad \mathrm{~m}_{2}=15 \\
\mathrm{~m}_{3}=4, \quad \mathrm{v}_{1}=0.25, \mathrm{v}_{2}=10, \gamma_{1}=10, \gamma_{2}=20, \gamma_{3}=8, \gamma_{4}=5, \gamma=15, \quad \mathrm{x}_{1}(0)=2.5 \\
\mathrm{x}_{2}(0)=0.25, x_{3}(0)=1.55, x_{4}(0)=1.35, \quad T=0.125
\end{gathered}
$$

1- The optimal diagrams obtained Pontryagin minimum principle: 

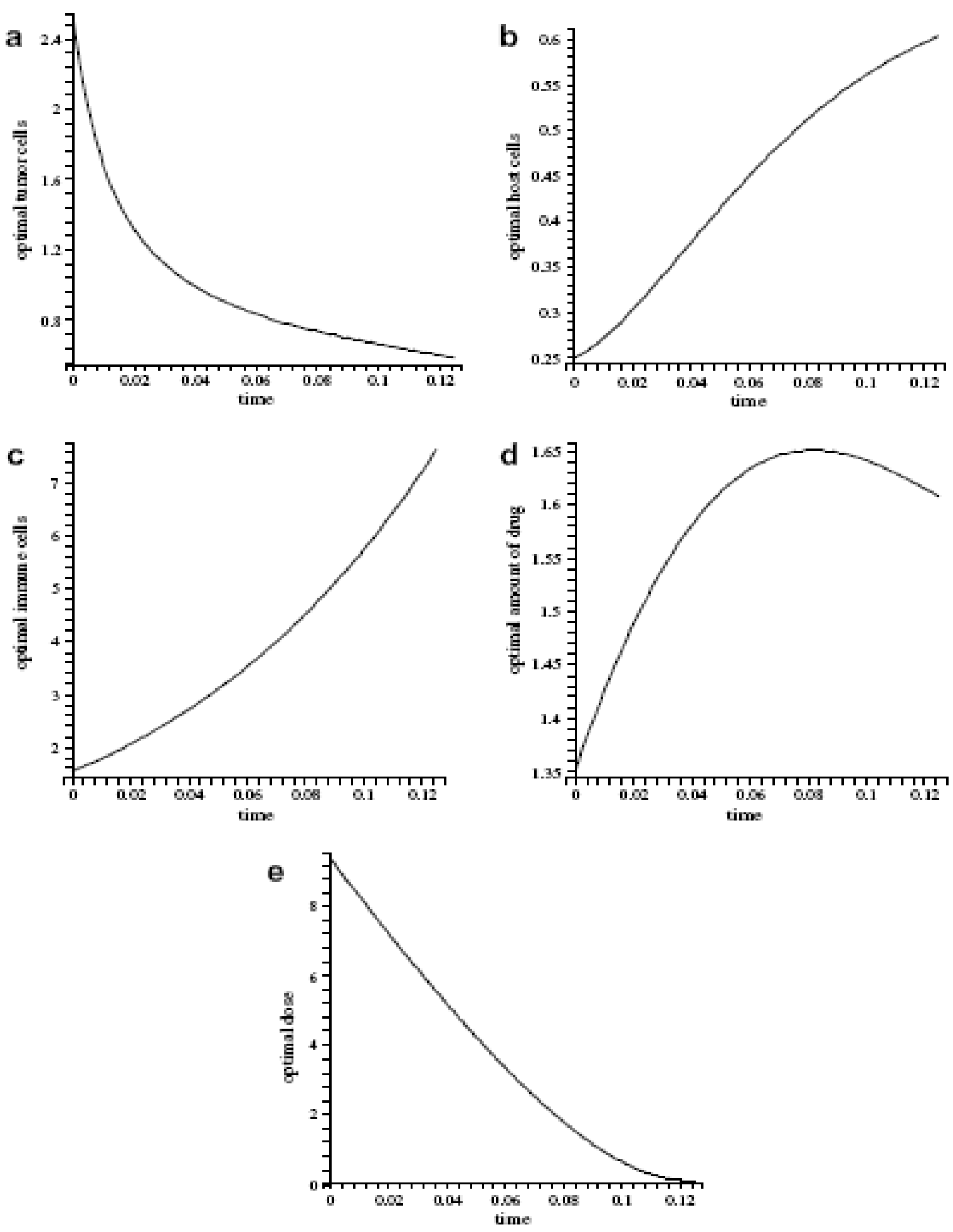

Fig. 1. (a) Optimal density of tumor cells. (b) Optimal density of host cells. (c) Optimal density of immune cells. (d) Optimal amount ofdrug. (e) Optimal dose of drug. 
2- The optimal diagrams obtained Measure Theory:
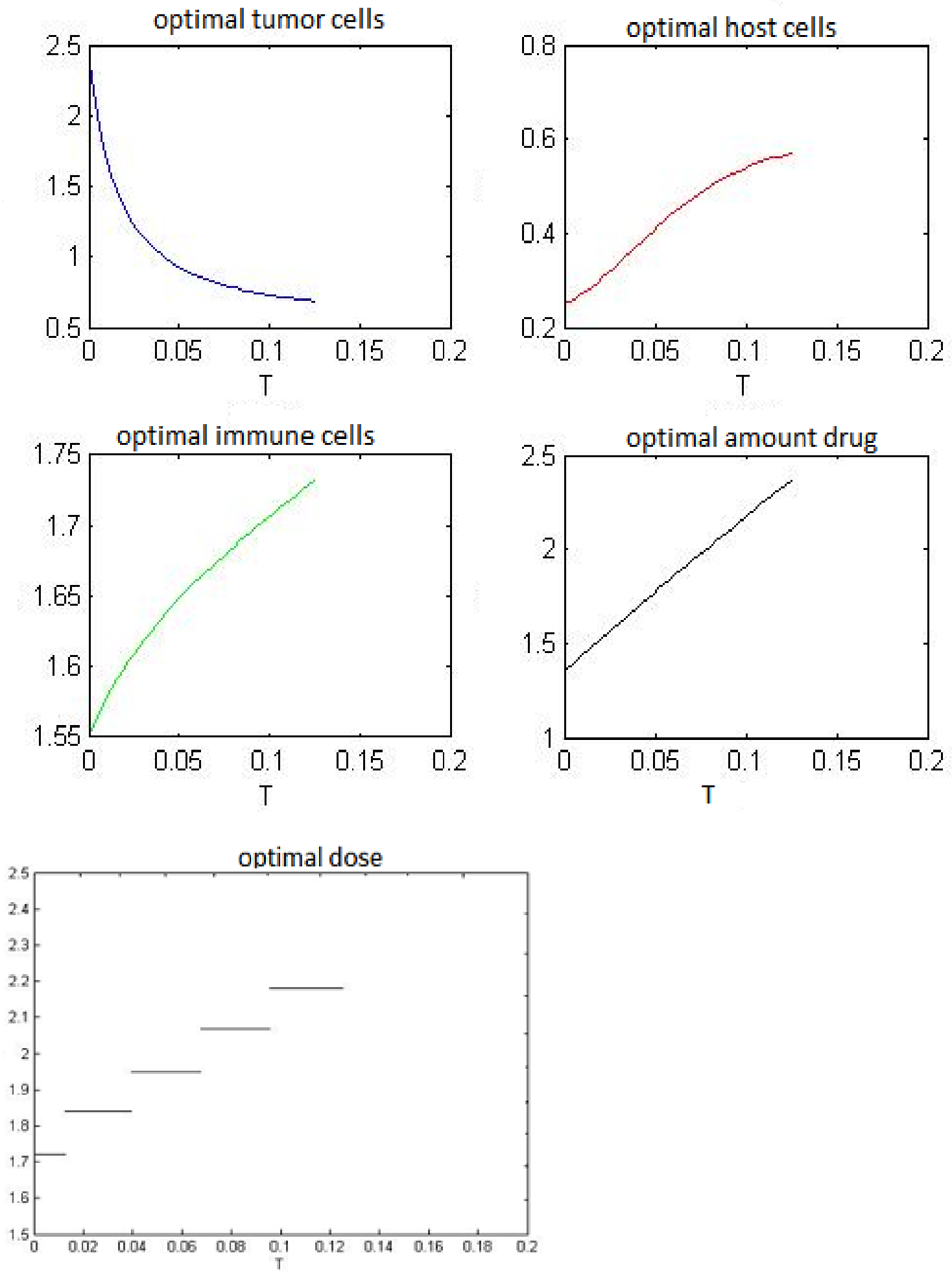

Fig. 2. (a) Optimal density of tumor cells. (b) Optimal density of host cells. (c) Optimal density of immune cells. (d) Optimal amount ofdrug. (e) Optimal dose of drug. 


\section{References}

[1]El-Gohary, A., Chaos and optimal control of equilibrium states of tumor system with drug, Chaos, Solitons\& Fractals, 2009; 14: 425-435.

[2]El-Gohary, A., chaos and optimal control of cancer self-remission and tumor system steady states, Chaos, solitons\& Fractals, 2008;37(5):1305-16.

[3]Dingli, D., Cascino, D., Josic, K., Russell, J., Bajzer, Z., Mathematical modling of cancer radiovirtherapy, Math Biosci, 2006:199:55-78.

[4]Mehmat,L., Metin U., Salamci, Stephan P.Banks, Optimal control of drug therapy in cancer treatment, Nonlinear Analysis 71, 2009; e1473-e1486.

[5]Kirschner, D., Panetta, J., modeling immunotherapy of tumor-immune interaction, J. Math.Biol., 1998;37(3):e235-52.

[6]Rubio, J. E., Control and Optimization: The Linear Treatment of nonlinear problems, Manchester Univercity Press,1986.

[7]Alavi, S.A., Kamyad, A.V., Farahi, M.H., Optimal control of an inhomogeneous heat problem by using measure theory, Iranian international journal science, 2000, 55-78. 